\title{
Temperature Homogenization of Co-Integrated Shape Memory - Silicon Bimorph Actuators
}

\author{
Gowtham Arivanandhan 1, Zixiong Li 1, Sabrina Curtis 2, Prasanth Velvaluri 2, Eckhard Quandt 2, \\ Manfred Kohl 1,* \\ 1 Institute of Microstructure Technology, Karlsruhe Institute of Technology (KIT), Karlsruhe 76131, \\ Germany; \\ 2 Institute for Materials Science, KielUniversity (CAU); Kiel 24143, Germany \\ * Corre spondence: manfred.kohl@kit.edu; Tel.: +49 721 608-22798
}

\begin{abstract}
The high work density and beneficial downscaling of shape memory alloy (SMA) actuation performance provide a basis for the development of actuators and systems at microscales. Here, we report a novel monolithic fabrication approach for the co-integration of SMA and Si microstructures to enableSMA-Si bimorph microactuation. Double-beam cantilevers are chosen for the actuator layout to enable electrothermal actuation by Joule heating. SMA materials under investigation are $\mathrm{NiMnGa}$ and $\mathrm{NiTi}(\mathrm{Hf})$ films with tunable phase transformation temperatures. We show that Joule heating of the cantilevers generates increasing temperature gradients for decreasing cantilever size, which hampers actuation performance. In order to cope with this problem, a new method for design optimization is presented based on FEM simulations. We demonstrate that temperature homogenization can be achieved by the design of additional folded beams in the perpendicular direction to the active beam cantilevers. Thereby, power consumption can be reduced by more than $35 \%$ and maximum deflection can be increased up to a factor of 2 depending on the cantilever geometry.
\end{abstract}

Keywords: microactuator, shape memory bimorph effect, finite element modeling, e-beam lithography, silicon technology

\section{Introduction}

Silicon (Si) technology has been well advanced to develop complex micro-electro-mechanical systems (MEMS) consisting of various functional units including structural parts, elastic components, as well as sensors and microactuators based on electrostatics, magnetomotive or electrothermal principles, see e.g. [1-3]. Despite this versatility, current microactuation concepts exhibit a number of limitations, including low pow er efficiency, limitations of downscaling, and technology constraints, which become even more severe upon further downscaling to nanometer dimensions. Shape Memory Alloy (SMA) based actuators may overcome these constraints due to their large w ork density of up to $10^{7} \mathrm{Jm}^{-3}$ and favorable downscaling behavior [4].

The monolithic integration of SMA actuators with active and passive Si structures enables various MEMS applications, including switching and tuning functions for mechanics and photonics $[5,6]$. The development of combined Si MEMS and SMA microactuator systems requires monolithic integration of SMA films compatible with MEMS fabrication processes. In recent years, monolithic fabrication as well as hybrid integration processes have been successfully demons trated, allowing for parallel fabrication of SMA film actuators on the wafer scale [7-9]. Major challenges are the high annealing temperature (minimum $500{ }^{\circ} \mathrm{C}$ ) and thermo-mechanical treatment required for SMA microactuator fabrication, which may cause interdiffusion, delamination, and process incompatibilities with other MEMS components [4]. Sputtered NiMnGa and TiNiHf SMA films are especially interesting for use in micro- and nanoactuators due to tunable phase transformation temperatures, tunablehysteresis, and decent functional fatigue properties [8-11]. 
SMA actuators being thermal actuators require either a direct or an indirect heating mechanism. Direct Joule heating can be implemented, in principle, down to nanometer dimensions, as it does not require any additional structures such as microheaters. However, direct Joule heating of SMA actuators consisting of beam cantilevers with decreasing dimensions results in an increasing temperature gradient along the beam length that can well exceed $50 \mathrm{~K} / \mu \mathrm{m}$ [12]. As a result, the SMA layer undergoes phase transformation only in a local region, which results in a negligible contribution of the shape memory effect to the deflection characteristics [12].

In this study, we present a process for co-integration of SMA and Si microstructures focusing on NiMnGa and NiTi(Hf) films to enable SMA/Si bimorph microactuation and introduce an approach to optimize the bimorph actuator layout with respect to temperature gradient in order to maximize deflection and to minimize power consumption.

\section{Fabrication of SMA-Si bimorph layer}

The process flow under investigation for the fabrication of SMA/Si bimorph microactuators is shown schematically in Figure 1. Double beam cantilevers are chosen for actuator layout to enable electro-thermal actuation by Joule heating. SOI wafers with Si device layer thickness of $2 \mu \mathrm{m}$ are chosen as the starting substrate for co-integration. SMA-based films are deposited by magnetron sputtering with a thickness of several microns along with a top layer of Au. Amorphous SMA films are then crystallized at selected temperatures $\left(500 \ldots 700^{\circ} \mathrm{C}\right)$. First, the pattern is transfer red to the Au layer by ultra-high-resolution electron beam lithography (EBL) and wet-etching of Au. Second, the thick SMA films are wet etched by hydrofluoric acid (HF) and nitric acid ( $\left.\mathrm{HNO}_{3}\right)$ solution, while thin films are dry-etched by reactive ion beam etching (RIBE). Third, the Si device layer is structured by reactive ion etching using the cryo process. Finally, freestanding SMA/Si bimorph cantilevers are obtained by wet etching of the buried silicon oxide using hydrofluoric acid. This proces slow is compatible with fabricating co-integrated SMA/Si bimorph and micromechanical structures. Figure 2(a) show s a scanning electron micrograph of a typical freestanding SMA/Si double-beam cantilever with a non-optimized layout. The SMA and Si layers of the bimorph device can be distinguished clearly.

(a)

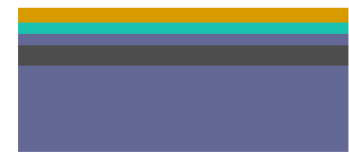

(d)

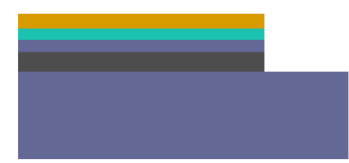

(b)

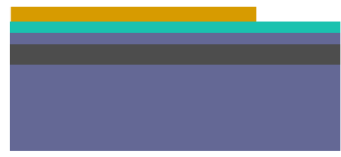

(e)

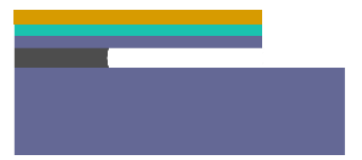

(c)

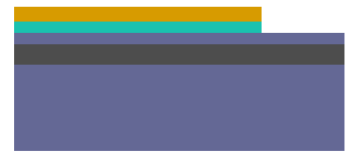

(f)

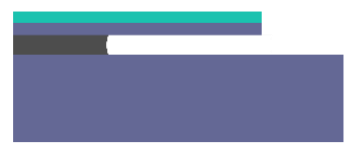

Gold

Figure 1. Schematic fabrication process flow of a SMA/Si bimorph microactuator: (a) SOI wafer with presputtered and annealed SMA and gold layer, (b) patterning of gold layer using EBL, (c) etching of SMA layer,

(d) RIE of Si de vice layer, (e) etching of $\mathrm{SiO}_{x}$ sacrificiallayer, (f) free-standing SMA/Si bimorph cantile ver.

\section{SMA Film Properties}

Energy dispersive x-ray spectroscopy (EDX) measurements show an average film composition of $\mathrm{Ti}_{38.9} \mathrm{Ni}_{48.8 \mathrm{Hf}} \mathrm{H}_{1.3}$ for sputtered amorphous TiNiHf films. Since the films are sputtered in the amor phous state, it is possible to adjust the SMA transformation temperatures (i.e actuator operation 
temperatures) via the annealing temperature. The Differential Scanning Calorimetry (DSC) measurements show $n$ in Figure 2(a) confirm that as the TiNiHf alloy annealing temperature increases, the transformation temperatures also increase. The latent heat, thermal hysteresis width, and transformation temperatures of the films annealed at $773 \mathrm{~K}, 873 \mathrm{~K}$ and $973 \mathrm{~K}$ for $30 \mathrm{~min}$ are given in Table 1 together with the phase transformation temperatures of a NiMnGa film. Figure 2(b) shows a DSC characteristic of the polycrystalline NiMnGa film, which has been heat-treated at $1073 \mathrm{~K}$. The chemical composition of the film has been determined by the inductive coupled plasma method to be Ni51.4Mn28.3Ga20.3.

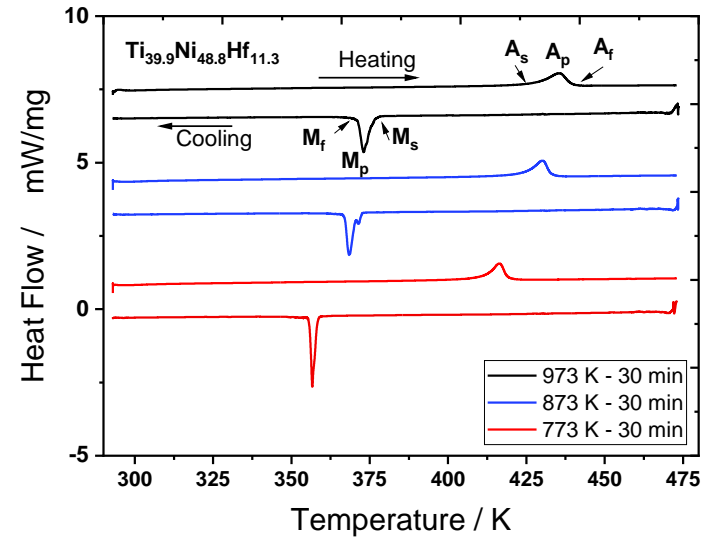

(a)

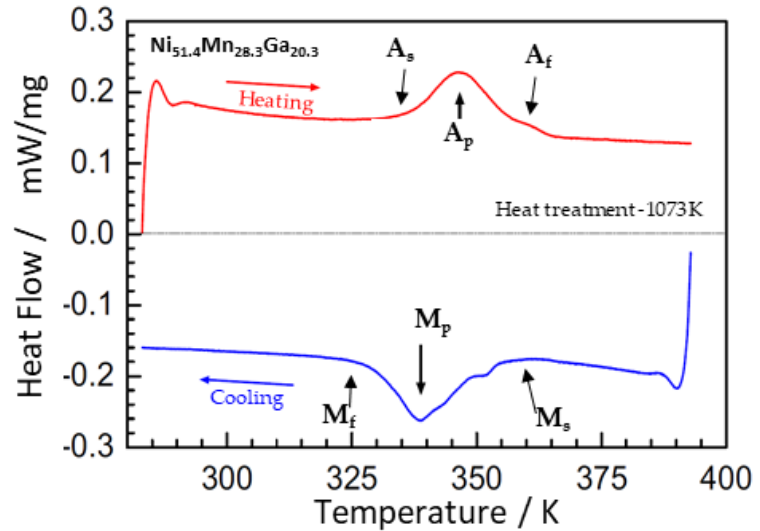

(b)

Figure 2. DSC characteristics of SMA thin films: (a) Influence of annealing on pha se transformation temperatures of TiNiHf films; (b) Phase transformation temperatures of a NiMnGa film.

Table 1. Differential scanning calorimetry data for sputtered TiNiHf and NiMnGa films after different annealing heat treatments.

\begin{tabular}{ccccccccc}
\hline \multirow{2}{*}{ Material } & $\begin{array}{c}\text { Annealing } \\
\text { Information }\end{array}$ & $\begin{array}{c}\text { Af } \\
(\mathbf{K})\end{array}$ & $\begin{array}{c}\text { As } \\
(\mathbf{K})\end{array}$ & $\begin{array}{c}\text { Ms } \\
(\mathbf{K})\end{array}$ & $\begin{array}{c}\text { Mf } \\
(\mathbf{K})\end{array}$ & $\begin{array}{c}\Delta \mathbf{T} \\
(\mathbf{K})\end{array}$ & $\begin{array}{c}\Delta \text { HM-A } \\
(\mathbf{J} / \mathbf{g})^{*}\end{array}$ & $\begin{array}{c}\Delta \text { HA-M } \\
(\mathbf{J} / \mathbf{g})^{*}\end{array}$ \\
\hline \multirow{2}{*}{ TiNiHf } & $773 \mathrm{~K}-30 \mathrm{~min}$ & 419.0 & 412.1 & 357.9 & 356.1 & 58.6 & 18.5 & 22.0 \\
& $873 \mathrm{~K}-30 \mathrm{~min}$ & 432.9 & 425.1 & 370.7 & 367.1 & 60.1 & 19.6 & 23.7 \\
& $973 \mathrm{~K}-30 \mathrm{~min}$ & 439.7 & 428.9 & 376.0 & 371.2 & 60.7 & 20.0 & 24.2 \\
\hline \multirow{2}{*}{ NiMnGa } & $1073 \mathrm{~K}-600 \mathrm{~min}$ & 355 & 337 & 360 & 326 & 3 & - & - \\
\hline
\end{tabular}

${ }^{*}$ Due to the low sample mass an error of $0.5 \mathrm{Jg}-1$ is assumed.

\section{Modelling Approach}

A Finite Element Model (FEM) for mechanical and thermal analyses is developed and implemented using COMSOL Multiphysics 5.5. The Electric-Current module, Heat-Transfer module and Structural-Mechanics module are coupled together to implement a Joule heating based deflection model of the SMA/Si bimorph devices. The resistive heating due to electro-thermal coupling acts as a thermal load for the Solid-Mechanics interface, causing differential thermal expansion as well as deformation due to martensitic phase transformation. 


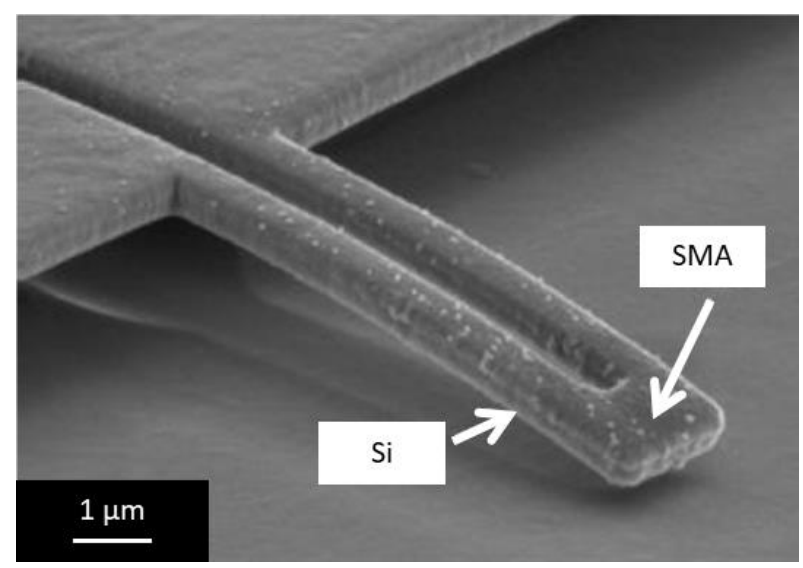

(a)

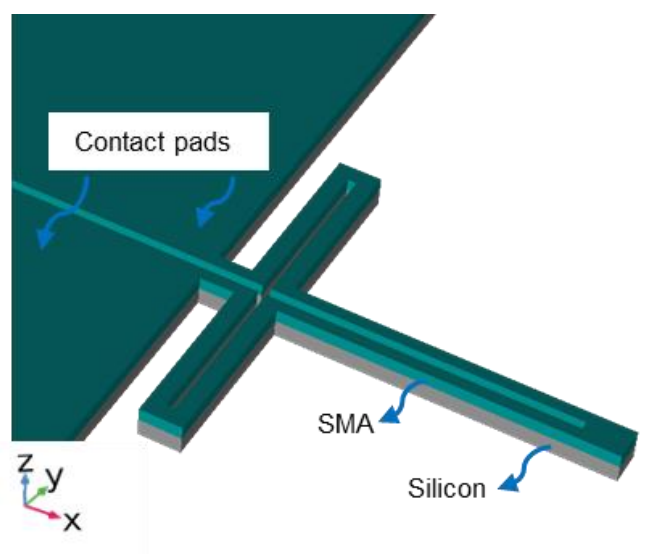

(b)

Figure 3. (a) Scanning electron micrograph of a freestanding SMA/Si bimorph microactuator with double-beam cantilever with the non-optimized layout; (b) schematic of a SMA/Si bimorph microactuator with additional folde d beam structures (wings) for temperature homogenization.

The shape memory effect is described by the Tanaka-type model, in which the martensite volume fraction $\xi_{M}(\sigma, T)$ is introduced that approximates the phase transformation by an exponential step function that varies between 1 (martensite state) and 0 (austenite state) depending on temperature and stress. In this phase transformation regime, the temperature-dependent material properties are defined by a simple rule of mixture, which introduces two independent parameter sets describing the martensite and austenite state. For example, the electrical conductivity $\beta_{S M A}(T)$ of SMA is approximated as a mixture of electrical conductivity in austenite phase $\beta_{A}$ and in martensite phase $\beta_{M}$,

$$
\beta_{S M A}(T)=\left(1-\xi_{M}(\alpha, T)\right) \cdot \beta_{A}+\xi_{M}(\alpha, T) \cdot \sigma_{M}
$$

Our approach for design optimization is to provide additional folded beam structures (wings) at the onset of the double-beam cantilever in a perpendicular direction as illustrated in Figure 3(b). In this case, the wing width, wing length, and the number of folds (overall path length) of the additional structures are the major design parameters for tuning the temperature gradient resulting from Joule heating.

Table 2 summarizes the material parameters of Si and two different SMA materials (NiMnGa and NiTi) used in the simulations. The key material parameters for resistive Joule heating are electrical conductivity $\beta$ and thermal conductivity $\kappa$. The thermal conductivity of SMA is approximated by the Wiedemann-Franz law [13] describing the heat conductivity $\kappa_{S M A}(T)$ as a function of electrical conductivity $\beta_{S M A}$,

$$
\kappa_{S M A}(T)=L_{0} \cdot \beta_{S M A}(T) \cdot T,
$$

with the proportionality constant $L_{0}=2.45 \times 10^{-8 W \Omega} / K^{2}$ [13]. The thermal conductivity of Si [14] is approximated by,

$$
\kappa_{S i}(T)=\frac{5.0105 \times 10^{4} \frac{W}{m}}{T}-19.24 \frac{W}{m K},
$$

For the mechanical response, the necessary input parameters are Young's modulus (E), Poisson ratio $(v)$, and the thermal expansion coefficient $(\alpha)$. In the phase transformation regime, the stress and the coefficient of thermal expansion (CTE) values are approximated by the rule of mixture as described before.

Table 2. Material parameters for coupled electro-thermo-mechanical simulation of temperature profiles for SMA/Si bimorph microactuators. Ma terial parameters of TiNiHf films a re a pproximated by parameters of binary 
TiNi films depending on data availability. The Poisson ratio of Si is included in the COMSOL material library. A polynomial function approximates the thermal expansion coefficient of Si [14] $\alpha_{S i}(T)=(-3.0451+$ $\left.0.035705 T-7.98110^{-5} T^{2}+9.578310^{-8} T^{3}-5.891910^{-11} T^{4}+1.461410^{-14} T^{5}\right) 10^{-6} 1 / \mathrm{K}$.

\begin{tabular}{cccc}
\hline & Si [15,16] & NiMnGa [17,18] & NiTi(Hf) [19,20] \\
\hline Electricalconductivity $\beta_{S M A^{\prime}}$ S/m & 10 & $\beta_{\text {NiMnGa }}(T)$ & $\beta_{\text {NiTi }}(T)$ \\
Thermalexpansion coefficient $\alpha, 1 / \mathrm{K}$ & $\alpha_{S i}(T)$ & $\alpha_{A}=23 \times 10^{-6}$ & $\alpha_{A}=11 \times 10^{-6}$ \\
Young's modulus E, GPa & 169 & $\alpha_{M}=33 \times 10^{-6}$ & $\alpha_{M}=6.6 \times 10^{-6}$ \\
Poisson's ratio $v$ & 0.22 & $\mathrm{E}=100$ & $\mathrm{E}=87$ \\
\hline
\end{tabular}

\section{Homogenization of Temperature Profiles}

Figure 4 show s a series of simulated temperature profiles of a NiMnGa/Si bimorph actuator for different values of electrical power. A standard double-beam cantilever design (denoted as nonoptimized design in the following) and a temperature-homogenized design with additional folded beam structure is considered with $20 \mu \mathrm{m}$ beam width and $200 \mu \mathrm{m}$ beam length. The width and length of the folded structures are 20 and $60 \mu \mathrm{m}$, respectively. In a first approach, a constant layer thickness of $2 \mu \mathrm{m}$ is considered for both SMA and Si layers. The x-position corresponds to the path along the beam length.

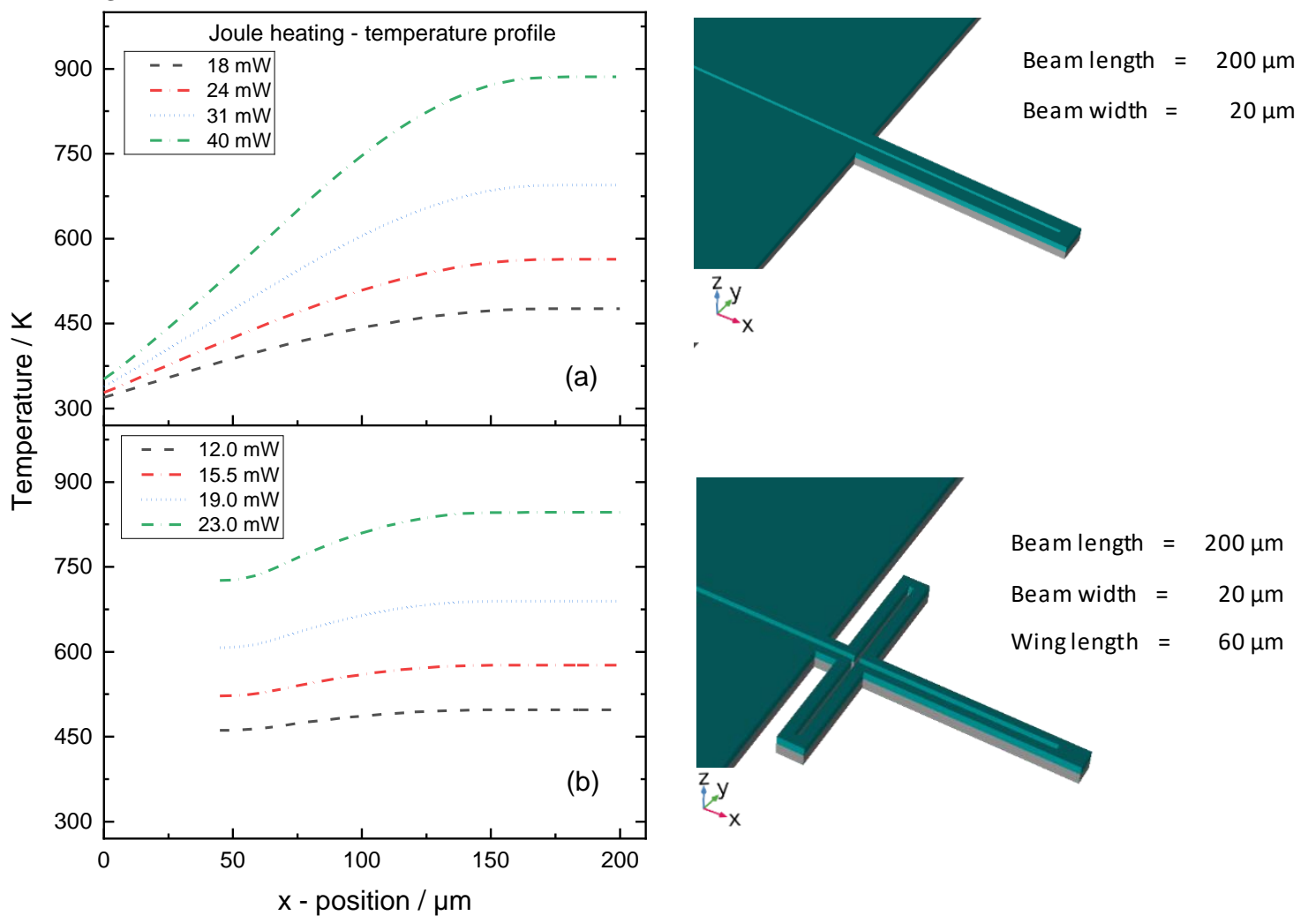

Figure 4. Coupled finite element simulation of temperature profile along $\mathrm{x}$-position of the beam for various values of electrical power as indicated. A double-beam cantilever with $20 \mu \mathrm{m}$ beam width and $200 \mu \mathrm{m}$ beam length is considered: (a) non-optimized design with very high temperature gradient, (b) temperaturehomogenized design with additional folded beam structure (wings). Less power is require do achieve similar maximum temperatures.

Figure 4(a) reveals that very high temperatures occur at the beam tips, while the onset of the beams is close to room temperature. At an electrical power of $40 \mathrm{~mW}$, the maximum temperature at the beam tip reaches $900 \mathrm{~K}$, while the beam onset has a temperature of $350 \mathrm{~K}$. As a result, a larger electric power is required to heat the entire structure, causing heat accumulation at the beam tip. 
Furthermore, for increasing electrical heating power, the temperature gradients along the beam lengths are increasing. Figure 4(b) shows temperature profiles of the temperature-homogenized design. Behind the wing structure $(x>50 \mu \mathrm{m})$, almost homogeneous temperature profiles occur along the beam in contrast to non-optimized cantilever structures. The homogeneous temperature profiles eliminate the issue of different phase states at local regions of the cantilevers and, thus, allow for complete phase transformation.

\section{Evaluation of Critical Electrical Power and Maximum Deflection}

At typical heat-treatment temperatures between $600 \mathrm{~K}$ and $800 \mathrm{~K}$, interdiffusion occurs and, consequently, shape memory properties deteriorate. Therefore, a critical temperature of $600 \mathrm{~K}$ is considered here as the operational limit of the actuator device. Figure 5(a) shows simulated characteristics of maximum temperature at the beam tip as a function of electrical heating power for different geometries of non-optimized and temperature-homogenized cantilevers. The critical electrical power limits of reaching the critical temperature of $600 \mathrm{~K}$ are indicated for both cases. The heating is maximum at the beam tip, so the beam tip is taken as the measurement point. The source of heat is characterized by the given electrical pow er. Therefore, the operational pow er limit is also calculated from this critical temperature, as indicated in Figure 5(a). For study about the dependencies of beam geometry, a set of different $\mathrm{NiMnGa} / \mathrm{Si}$ bimorph variants is selected featuring beam widths and lengths up to $30 \mu \mathrm{m}$ and $200 \mu \mathrm{m}$ (see Table 3).

Table 3. Geome tric dimensions of different variants of NiMnGa/Sibimorph actuators

\begin{tabular}{cccc}
\hline Variant & Beam width, w $(\boldsymbol{\mu m})$ & Beam length, $\mathbf{l}(\boldsymbol{\mu m})$ & Wing length $^{\mathrm{a}}, \mathbf{h}(\boldsymbol{\mu m})$ \\
\hline 1 & 2 & 20 & 5 \\
2 & 2 & 100 & 15 \\
3 & 4 & 60 & 15 \\
4 & 20 & 200 & 60 \\
5 & 30 & 200 & 60 \\
\hline
\end{tabular}

a Only for the folded beam structure; the wing width always coincides with the beam width of the cantilever

Comparing variants $\# 1$ and \#4, the width and length are upscaled by a factor of 10 while keeping the width-to-length ratio constant ( $\mathrm{w} / \mathrm{l}$ aspect ratio of 10$)$. The critical electrical pow er for variants \#1 and \#4 for the non-homogenized model is $19.7 \mathrm{~mW}$ and $26 \mathrm{~mW}$, respectively. In the case of the temperature-homogenized model, this critical power limit decreases to $16 \mathrm{~mW}$ and $12.5 \mathrm{~mW}$, which corresponds to a $35 \%$ reduction in power consumption. Comparing variants $\# 4$ and $\# 5$ in Figure $5(\mathrm{a})$, a huge difference of $40 \%$ in critical power is observed, resulting from the variation of beam width. In the case of $100 \mu \mathrm{m}$ beam length, both models reveal the low est values of critical pow er of $4.5 \mathrm{~mW}$ (non-optimized) and $3.5 \mathrm{~mW}$ (temperature-homogenized), respectively. In all cases, temperaturehomogenized designs reveal lower electrical pow er to achieve comparable maximum temperatures. 


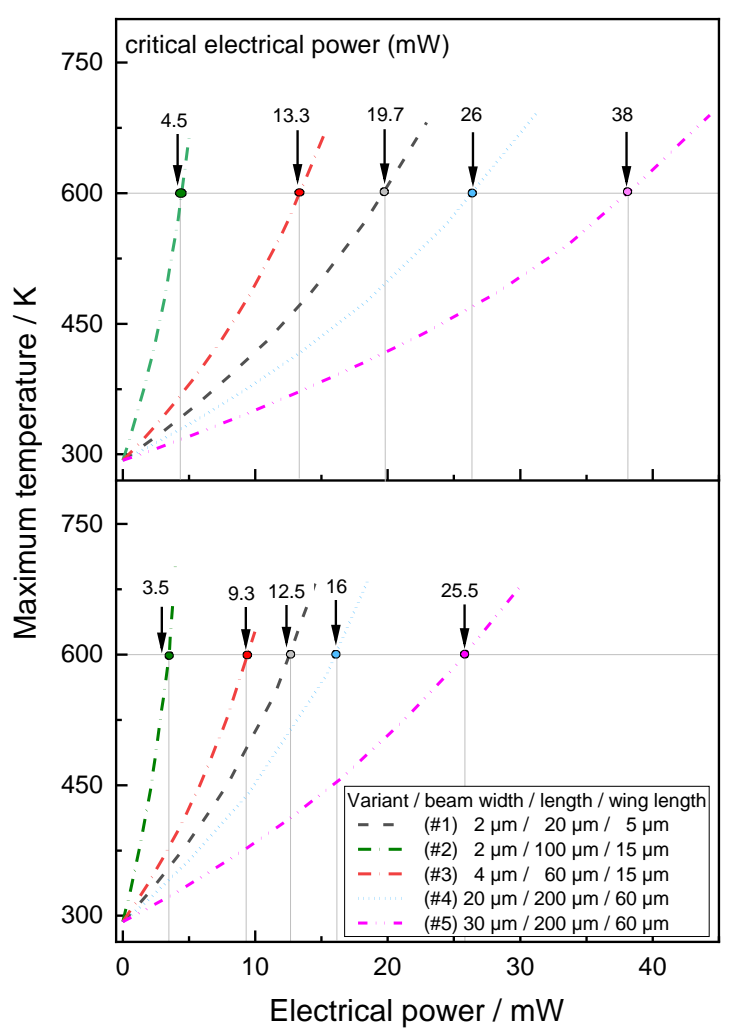

(a)

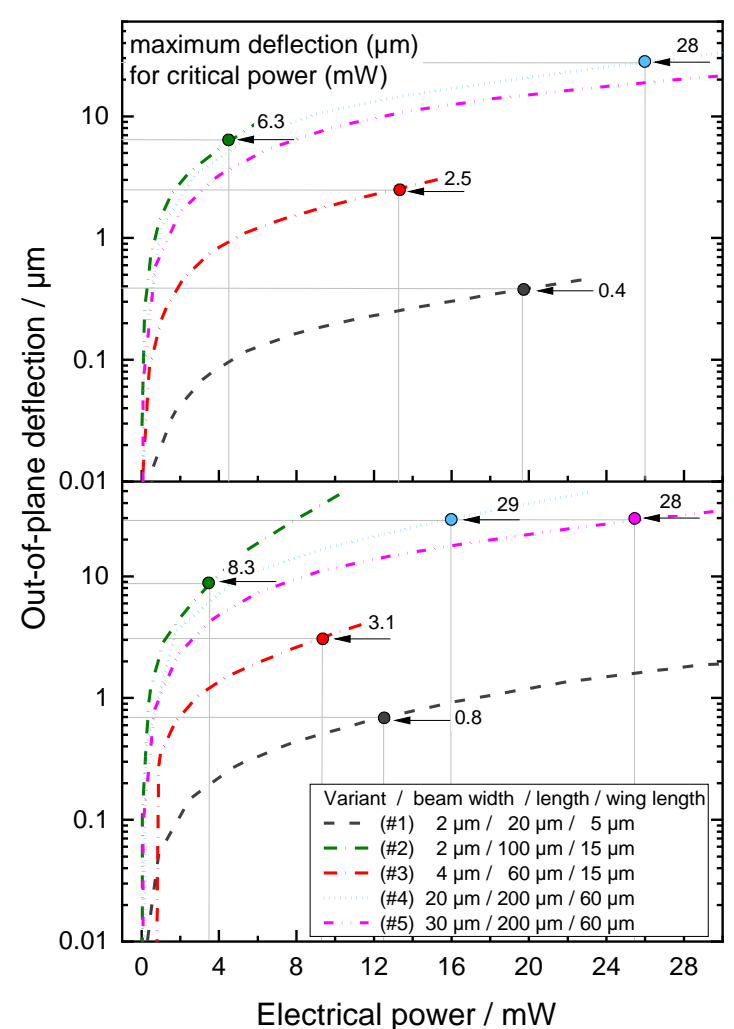

(b)

Figure 5. (a) Simula ted cha racteris tics of maximum temperature at the beam tip as a function of electrical heating power for different geometries of non-optimized and temperature-homogenized cantilevers. The critical electrical power limits of reaching the critical temperature of $600 \mathrm{~K}$ are indicated for both cases. (b) Simulated characteristics of out-of-plane deflection at the beam tip as a function of electrical heating power. The maximum achievable deflections a re indica te d corresponding to the critical electrical power limits.

Simulated characteristics of out-of-plane deflection at the beam tip are shown in Figure 5(b) as a function of electrical heating power. The maximum achievable deflections are indicated corresponding to the critical electrical pow er limits. Comparing variant \#1 of both the non-optimized and temperature-homogenized designs, the deflection increases by a factor of 2, and power consumption reduces by more than $35 \%$. In general, for bimorphs, the thickness of SMA/Si layers and the length of the cantilever defines the out-of-plane deflection, whereas the beam width has no contribution to it. This is observed by comparing variants $\# 4$ and $\# 5$, where the deflection is merely the same for both non-homogenized and homogenized models. How ever, a significant reduction of the critical power limit by $30-40 \%$ is present. This implies that the range of power limit can be changed without compromising the deflection, depending on the design requirements.

\section{Conclusions}

This study presents an approach to fabricate SMA/Si bimorph microactuators with cantilever beam design and to homogenize their temperature profile caused by Joule heating. The focus is on $\mathrm{NiMnGa}$ and $\mathrm{NiTi}(\mathrm{Hf})$ films with tunable phase transformation temperatures. By introducing folded beam structures perpendicular to the cantilever structures, the temperature profiles are homogenized, allowing for uniform phase transformation. Critical electrical power values are determined for different geometric variants indicating the operational limit of the SMA/Si bimorph microactuators. By temperature homogenization, we could reduce pow er consumption by up to $35 \%$. Upscaling the temperature-homogenized geometry without changing the aspect ratio $\mathrm{w} / \mathrm{l}$ allows for reduction of electrical heating pow er by $30-40 \%$. The maximum deflection is increased by a factor of 2 for the smallest cantilever design under investigation with beam width/length of $2 / 20 \mu \mathrm{m}$. 
Acknowledgments: This research has received funding from the German Research Foundation (DFG) within the priority program "SPP2206 - Cooperative Multistage Multistable Microactuator Systems". Further, we would like to thankDuygu Dengiz for supporting us with EDX measurements.

Author Contributions: M.K., and E.Q. developed the concept; G.A. de veloped the simula tion model, performed the simulations and experiments, analyzed the data and wrote the draft of the manuscript;Z.L. contributed to experiments and data analysis; S.C designed the TiNiHf experiments and analyzed the data; P.V. contributed to SMA sample pre paration; M.K. supervised the work, re vie wed and edited the manuscript.

Conflicts of Interest: The authors declare no conflict of interest

\section{Abbreviations}

The following abbreviations are used in this manuscript:

EBL: Electron Beam Lithography

EDX: Energy dispersive x-ray spectroscopy

CTE: Coefficient of Thermal Expansion

DSC: DifferentialScanning Calorimetry

FEM: Finite Element Modeling

MEMS: Micro-Electro-MechanicalSystems

RIBE: Reactive Ion Beam Etching

SMA: Shape Memory Alloy

SOI: Silicon-On-Insulator

\section{References}

[1]. S. Hollar, S. Bergbreiter, K.S.J. Pister. (2003) Bi-directional inchworm motors and two DOF robot leg operation. Proc. Transducers'03; DOI: 10.1109/SENSOR.2003.1215303.

[2]. M. Last, V.Subramaniam, K.S.J. Pister. (2005) Out-of-plane motion of a ssemble d microstructures using a single-maskSOI process. Proc. Transducers Seoul Korea; doi: 10.1109/SENSOR.2005.1496509

[3]. R. Yeh, S. Hollar, and K. S. J. Pister. (2001). Single mask, la rge force, and large dis placement electrostatic line ar inchworm motors. Technical Digest. MEMS 2001. 14th IEEE International Conference on Micro Electro Mechanical Systems (Cat. No.01CH37090). pp. 260-264, doi: 10.1109/MEMSYS.2001.906528.

[4]. M. Kohl, Shape Memory Microactuators. Berlin Heidelberg: Springer-Verlag, 2004.

[5]. F. Lambrecht, I. Aseguinolaza, V. Chernenko, and M. Kohl. (2016). Integrated SMA-based NEMS actuator for optical switching. IEEE 29th International Conference on Micro Electro Mechanical Systems (MEMS). pp. 79-82; doi: 10.1109/MEMSYS.2016.7421562.

[6]. R. Fechner, C. Chlub, E. Quandt, and M. Kohl. (2018) .A Shape Memory Alloy 1×2 Optical Waveguide Switch. IEEE 18th International Conference on Nanotechnology (IEEE-NANO). pp. 1-3; doi: 10.1109/NANO.2018.8626320.

[7]. S. Braun, N. Sandstrom, G. Stemme, and W. van der Wijnga art. (2009). Wafer-Scale Manufacturing of Bulk Shape-Memory-Alloy Microactuators Based on Adhesive Bonding of Titanium-Nickel Sheets to Structured Silicon Wafers. Journal of Microelectromechanical Systems, vol. 18, no. 6, pp. 1309-1317; doi: 10.1109/JMEMS.2009.2035368.

[8]. F. Lambrecht, N. Sagardiluz, M. Gueltig, I. R. Aseguinolaza, V. A. Chernenko, and M. Kohl. (2017). Martensitic transformation in NiMnGa/Si bimorph nanoactuators with ultra-low hysteresis. Applied Physics Letters, vol. 110, no. 21, p. 213104; doi: 10.1063/1.4984058.

[9]. M. Kohl, M. Schmitt, A. Backen, L. Schultz, B. Krevet, and S. Fähler. (2014). Ni-Mn-Ga shape memory nanoactuation. Applied Physics Letters, vol. 104, no. 4, p. 043111; doi: $\underline{10.1063 / 1.4863667}$. 
[10].Karaca, H. E., Acar, E., Tobe, H., \& Saghaian, S. M. (2014). NiTiHf-based sha pe memory alloys. Materials Science and Technology, 30(13), 1530-1544.

[11].Bechtold, C., Chluba, C., Zamponi, C., Quandt, E., \& de Miranda, R. L. (2019). Fabrication and Characterization of Freestanding NiTi Based Thin Film Materials for Shape Memory Micro-actuator Applications. Shape Memory and Superelasticity, 5(4), 327-335.

[12].F. Lambrecht, C. Lay, R. Aseguinolaza, V. Chernenko, and M. Kohl. (2016). NiMnGa/Si Sha pe Memory Bimorph Nanoactuation. Shape Mem. Superelasticity 2, 347-359.

[13].K. Kopitzki and P. Herzog. (2004). Einführung in die Festkörperphysik, 5. Teubner, Auflage.

[14].H. Watanabe, N. Yamada, and M. Okaji. (2004). Linear Thermal Expansion Coefficient of Silicon from 293 to 1000 K. International Journal of Thermophysics, vol. 25, no. 1, pp. 221-236; doi: 10.1023/B:IJOT.0000022336.83719.43.

[15].Jeung-hyun Jeong; Sung-hoon Chung; Se-Ho Lee; Dongil Kwon. (2003). Evalua tion of Ela stic Properties and Temperature Effects in Si Thin Films Using an Electrostatic Microresonator. Journal of Microelectromechanical Systems, vol. 12, no.4, pp. 524-530; DOI: 10.1109/JMEMS.2003.811733

[16].J. A. Carruthers, T. H. Geballe, H. M. Rosenberg, and J. M. Ziman. (1957). The ThermalConductivity of Germanium and Silicon between 2 and $300^{\circ} \mathrm{K}$. Proceedings of the Royal Society of London. Series A, Mathematical and Physical Sciences, vol. 238, no. 1215, pp. 502-514, 1957.

[17].I. R. Aseguinolaza, I. Orue, A.V.Svalov, K.Wilson, P.Müllner, J.M. Barandiarán, V.A.Chernenk (2014). Martensitic transformation in Ni-Mn-Ga/Si(100) thin films. Thin Solid Films, vol. 558, pp. 449-454; doi: 10.1016/j.tsf.2014.02.056.

[18].I. R. Aseguinolaza, I.R. Salazar, A.V. Svalov, K.Wilson, W.B.Knowlton, P.Müllner, J.M. Barandia rán, V.A.Chernenk. (2012). Transformation volume strain in Ni-Mn-Ga thin films. Applied Physics Letters, vol. 101, no. 24, p. 241912; doi: 10.1063/1.4772005.

[19].O. Benafan. (2012). Deformation and phase transformation processes in polycrystalline NiTi and NiTiHf high temperature shape memory alloys. Ph.D. the sis, University of Central Florida, Florida.

[20]. P. Velvaluri, M. S. Pravdivtseva, R. Lima de Miranda, J. B. Hövener, O. Jansen, and E. Quandt. (2019). Design Characterization of Thin Film Flow Diverter Stents (FDS) Based on SMA's: FEA, CFD and MRI Study. Shap. Mem. Superelasticity, vol. 5, no.3, pp. 195-205; doi: 10.1007/s40830-019-00223-8. 